\title{
Los estudios teóricos de la literatura y la necesidad de la interpretación
}

Margarita Rojas G.

Universidad Nacional, Costa Rica

1. Si uno quiere preparar unos chicharrones para invitar a unos amigos a tomarse unas cervezas un sábado en la noche, ¿cuál de los siguientes libros utilizaría?:

[a] un tratado de química sobre la estructura molecular de las sustancias lípidas, sus características, la forma de combinación, punto de ebullición;

[b] un libro de nutrición sobre las grasas, sus beneficios y problemas, necesidades según edades, tipos de enfermedades; [c] una historia de la aparición en Occidente de la dieta con carne de cerdo, sus formas de cocción en la antigua Roma, el paso a América, la incorporación a la dieta náhuatl en Mesoamérica;

[d] la Cocina práctica, de la tía Florita, con varias formas de preparar la came de cerdo, su presentación y posibles ingredientes.

Con el libro de química seguramente hablaríamos de lípidos, con el de nutrición sobre grasas y colesterol, la Historia probablemente nos remontaría al cerdo y sus antepasados salvajes; el recetario, por último, nos enfrentaría más directamente con la receta de la carne de chancho.

Creo que en la práctica de los estudios literarios utilizamos todos los libros no importa para qué y a veces nos encontramos preparando 
bocas de chicharrón con una investigación sobre los lípidos. Y me parece que el problema va más allá de un asunto de semántica. Por lo que se puede leer en las publicaciones de varios países el problema desborda las fronteras nacionales. Se trata, aparentemente, de una polémica en el ámbito internacional que nos debería permitir aquí iniciar una reflexión colectiva para saber si en realidad todos estamos trabajando en lo mismo o, por el contrario, algunos están estudiando los lípidos, otros la grasa o el cerdo y tal vez algunos están preparando un buen plato de chicharrones.

El punto principal de este trabajo es la necesidad de distinguir la investigación teórica de la literatura de su interpretación; para ello, se hará una referencia sintética al debate entre los pragmatistas, los desconstructivistas y otros teóricos, así como a la distinción entre teoría y hermenéutica. Para ello se partirá de una reflexión sobre los tipos de enfoques académicos más comunes.

2. Leyendo un número reciente de una revista mexicana de semiótica greimasiana, en la sección titulada "semiótica y estética", se esperaría que los artículos se consagren a estudios propios de la disciplina, por ejemplo, algún aspecto de la teoría de las modalidades o de las pasiones. En su lugar, los dos primeros trabajos se dedican al estudio, a la interpretación de textos particulares - una novela, Facundo, un poema de Octavio Paz-y las conclusiones se refieren, igualmente, a los textos, y no al supuesto problema teórico que se planteaba en el inicio.

En un reciente congreso fuera de Costa Rica, muchas ponencias insistían en analizar diversos textos intercalando repetidas citas de la teoría psicoanalítica de Lacan. Algo semejante sucede con Kristeva, Bajtin, etc., que era lo mismo que ocurría veinte años atrás con Genette, Todorov, Barthes. Y a veces el problema se duplica, porque se cita a partir de fuentes de segunda mano, resúmenes de diccionarios, apuntes, citas, un autor referido por otro.

Por otro lado, sorprende el libro de Greimas, De la imperfección (1987). Se trata de unos ensayos literarios sobre algunos textos: un 
poema japonés y uno de Rainer María Rilke, "Continuidad de los parques" de Cortázar y otros. Desde el título, la presentación y la nota final, el libro se centra en la búsqueda del sentido como algo escondido, "Querer decir lo indecible, tomar lo invisible: (...) pruebas de que algo puede ser posible" (99), el parecer que esconde el ser, la idea de la imperfección: "Todo parecer es imperfecto: esconde el ser y a partir suyo se construye un querer-ser y un deber-ser, lo que es una desviación del sentido. Sólo el parecer (...) es apenas soportable" (9). Pareciera el desahogo después de una vida dedicada al mayor esfuerzo de formalización de la semiótica del siglo xx, que, sin embargo, reconoce no sólo la existencia sino también lo placentero de la actividad de la interpretación y su escritura.

¿Qué es lo que sucede? Lo que hallamos en la revista de semiótica mexicana se repite por doquier: la persistencia del interés por la interpretación, disfrazada o legitimada por fachadas teóricas, más o menos aludidas al inicio y en algunas notas al pie de página. Todo muestra la preocupación subterránea por responder a una necesidad constante: ¿Qué significa este texto? El asunto ahora es entender si esto está bien o está mal, si se trata simplemente del fracaso del proyecto teórico, de una mala lectura de sus metas, un fraude, un engaño, una pose intelectual, o bien, la permanente y vergonzante preocupación de ciudadanos del tercer mundo por mostrar estar al día con las teorías europeas.

Dejando a un lado el trabajo crítico y filológico, podríamos empezar a distinguir tres tipos de posiciones en los estudios de la literatura en la práctica académica en Costa Rica:

a. la histórica, que consiste en la "aplicación" sobre el texto de rasgos ya codificados por las historias de la literatura. Se trata de una práctica muy común en el estudio de la literatura en la secundaria: se pide al estudiante que determine tres características del modernismo en un poema, o bien, que explique por qué un cuento puede considerarse romántico, etc. Este tipo de 
ejercicios tienen cierta utilidad pero no resuelven el problema de la significación del texto: descubrir o reafirmar que una metáfora de El Señor Presidente es surrealista no revela sus significados;

b. la temática, que estudia un tema seleccionado de antemano. En esta perspectiva se podrían incluir los llamados 'estudios culturales', los poscoloniales, los feministas, etc., que cada cierto tiempo aparecen en los Estados Unidos y cuyos ecos también se escuchan por acá. Este tipo de trabajos tampoco resuelve el problema de la interpretación, porque se procede aislando un elemento del texto. Con esto se pierde la necesaria articulación de la estructura mayor y no se puede alcanzar la significación global;

c. la "aplicación" de una teoría o parte de un modelo teórico como análisis del texto. Se toma una idea de Genette, Bajtin o Lacan y se establece una suerte de correspondencia con una novela, un poema o un cuento. Surgen así una multitud de tesis y ponencias tituladas "El cronotopo en la Caña brava de Luis Dobles Segreda", "En una silla de ruedas: novela polifónica", "El estadio del espejo en El túnel o en Los cachorros". En este tercer caso, los resultados no conducen a un trabajo teórico, porque generalmente no se realiza ningún aporte original con respecto a las teorías referidas, ni tampoco se llega a una interpretación cabal del texto, porque en la "aplicación" de las teorías se pierden las cualidades específicas del material analizado, al enfocar el estudio únicamente en los conceptos teóricos tomados en préstamo.

Pero también se corre otro riesgo y es que muchas veces el hecho de volver a hallar en el texto el modelo teórico o uno de sus rasgos hace que el estudio se presente como si hubiera conducido a la comprensión del texto, de su significación: se cree o se trata de hacer creer que, el encontrar que en la novela $X$ algo que había determinado Bajtin, o bien, que se trata de novela histórica o un cuento fantástico porque se hallaron los rasgos señalados por Seymour Menton o por Tzvetan 
Todorov, consiste en haber encontrado la estructura significativa de esa novela.

En cualquiera de los tres casos algunas veces el trabajo puede conducir a resultados interesantes, generalmente cuando cambia la intención primera. Pero en el sentido original, las "aplicaciones" de teorías, la búsqueda de rasgos históricos o el rastreo de temas, son prácticas altamente redundantes porque, o describen lo ya leído en el texto analizado, o bien, repiten las nociones tomadas de los marcos teóricos o históricos. Como había advertido Greimas en 1976, son "técnicas repetitivas, sin proyecto científico", "aplicaciones mecánicas de esquema" que no consiguen un aumento real del conocimiento de las organizaciones textuales y tampoco sirven para dar cuenta de la especificidad de los textos estudiados ${ }^{1}$.

3. Parece que la cuestión podría considerarse por lo menos desde tres perspectivas: una, más propia de los años setenta, que se debate dentro del antagonismo del tercer mundo con el primero y que a veces proclama, ingenuamente, la necesidad de abandonar toda teoría europea a cambio de teorías y categorías producidas en Latinoamérica. Todavía se perciben ecos de esta posición en algunos planteamientos como los de Homi Bhabha en Inglaterra y los llamados poscolonialistas.

El problema se podría enfrentar también desde una perspectiva histórica, a partir de la ruptura con la filología y la historia de la literatura provocada por el surgimiento del estructuralismo, en los años sesenta, que generó también un rechazo a todo proyecto de interpretación. Pues uno de los postulados fundamentales de todos los estructuralismos - genéticos, sociológicos, etc.—y también de la semiótica, era estudiar los mecanismos de producción de sentido y esto significaba el abandono de toda pretensión de establecer esos sentidos en el texto. Es decir, paradójicamente se proponía el estudio de los artificios textuales que generaban algo que se prohibía estudiar.

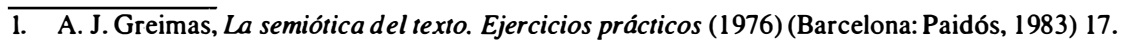


Lo anterior condujo a la ignorancia sistemática de la comunidad interpretativa: se incluía dentro del término despectivo de la "crítica", todo intento de exégesis y lecturas simbólicas, fenomenológicas, etc., que adquirieron connotaciones negativas, censuradas, prohibidas. Así, la revisión bibliográfica o el estado de la cuestión de toda tesis, por ejemplo, tenía que condenar de antemano todo análisis anterior y, en la práctica, se partía de cero, sin tomar en consideración contribuciones precedentes. Todas estas eran denominadas "la crítica", sin distinción posible, lo cual, con la distancia temporal de la actualidad permite apreciar una primera confusión: ignorar la diferencia entre la crítica como el dictamen valorativo sobre la calidad de una obra, el juicio estético, y la interpretación comoel trabajo con la estructura semántica del texto.

Se confundieron crítica e interpretación, se relegaron la historia y la filología, y el oficio con la literatura se fragmentó en muchas áreas de trabajo que sobrevivieron ignorándose. El legado actual es un amplio mapa en el que, tomando en cuenta únicamente el siglo $\mathrm{xx}$ y dejando por fuera la crítica y la historia, hay que reconocer por lo menos nueve disciplinas interpretativas y de análisis formal, siete versiones del estructuralismo, cuatro orientaciones semióticas, la narratología de Gérard Genette y dos posiciones de la estilística.

La mayoría de esas teorías, "las más influyentes actualmente son antiintencionalistas", se señala en el segundo Nuevo diccionariodelas ciencias del lenguaje, publicado hace pocos años, por los lingüistas Ducrot y Schaeffer ${ }^{2}$. Esto significa que niegan la pertinencia o la necesidad de identificar la significación textual, la intención en acto, la encarnada en el texto —que no se debe confundir con la intención previa o intención del autor-.

Entonces, además de la distinta afiliación filosófica, los modelos teóricos se distinguen por el grado de antiintencionalismo: desde los menos radicales, que reducen la intencionalidad de superficie a

2. Oswald Ducrot y Jean Marie Schaeffer, Nuevo diccionario de las ciencias del lenguaje (1995) (Madrid: Arrecife, 1998) 188. 
representaciones inconscientes subyacentes o a una simple expresión de factores causales no intencionales, por ejemplo, los análisis psicoanalíticos, los análisis ideológicos y las teorías marxistas (que remiten las estructuras discursivas a una voluntad de poder o estrategias de clase), hasta el antiintencionalismo radical, propio de Derrida y el desconstruccionismo, que niegan la pertinencia como tal de la noción de intencionalidad.

Como se señala en este Diccionario, el problema surge porque, al mismo tiempo que existe esta primacía de los estudios antiintencionalistas, no se puede ignorar que "la obra literaria es un fenómeno intencional"3. Por lo tanto, y este me parece que es un importante señalamiento, se reconoce que la intencionalidad es "el talón de Aquiles" de los estudios literarios, ya que todo estudio de la literatura pasa necesariamente por la práctica interpretativa, pues su materia es un conjunto de discursos. Esta importante manifestación seguramente es un ingrediente más en la polémica contra el desconstruccionismo y el pragmatismo y también justifica necesarias aclaraciones sobre el lugar del trabajo teórico.

4. El problema que se plantea entonces consiste básicamente en discutir la necesidad de la interpretación y distinguirla del proyecto teórico. Muchos son los signos de la importancia del tema de la interpretación en la última década del siglo xx; Umberto Eco habla de una batalla teórica por una redefinición del papel de la interpretación ${ }^{4}$. En el ámbito latinoamericano, se pueden citar los tres artículosreseñas dedicados a la hermenéutica de acuerdo con Heidegger y Gadamer publicados en el número 6 del año 2000 de la revista Semiosis de la Universidad Veracruzana; también en México había aparecido un poco antes Teoría del texto e interpretación de textos, de Walter Mignolo. En el ámbito europeo, recordemos la edición de 1995

3. Ducrot, 190.

4. Umberto Eco, Interpretación y sobreinterpretación (1992) (Cambridge: Cambridge University Press, 1995) 23. 
de los trabajos sobre hermenéutica de Hans Georg Gadamer; más recientemente Tzvetan Todorov dedica un capítulo del libro El hombre desplazado a la crítica de los estudios de la literatura en EE. UU. Uno de los más activos en la polémica ha sido Umberto Eco, cuya posición se puede apreciar en el tomo Los límites de la interpretación, de 1990; sus conferencias y el debate posterior con algunos desconstruccionistas en Inglaterra, en 1990 fueron recogidos en el libro Interpretación y sobreinterpretación, edición en español de 1995. Este año apareció también la edición en francés del ya referido Nuevo diccionario de las ciencias del lenguaje, editado por Oswald Ducrot esta vez con Jean Marie Schaeffer.

5. Relativismo. Un punto central del debate ha sido la idea de que un texto, bien por su polisemia, o bien, por la interacción con el Lector, genera múltiples sentidos $\mathrm{y}$, por lo tanto, presenta varias interpretaciones. A esta idea se ha tratado de encontrar fundamento, con poca exactitud aunque con amplia difusión, en la noción de la "semiosis ilimitada", propuesta por Charles S. Peirce a principios del siglo $\mathrm{xx}$.

La significación tiene un carácter indeterminado, pues lo único real es la circulación infinita de los signos; por este motivo, la relación de interpretación siempre se vuelve a lanzar, el sentido siempre es rechazado y, en consecuencia, no vale la pena realizar el esfuerzo de analizar un texto.

Sin embargo, es muy común encontrarse con la referencia a Derrida en trabajos que buscan interpretar un texto o, como se dice a veces, "deconstruir" un texto, que a fin de cuentas viene a ser lo mismo. Las lecturas parciales de un texto se defienden entonces a partir de la idea de la infinitud de posibilidades de interpretación de todo texto y, por lo tanto, se elimina la polémica para defender una de todas las posibles "lecturas", ya que todas, de acuerdo con Derrida, resultarían igualmente válidas.

5. Hans Georg Gadamer, El giro hermenéutico (1995) (Madrid: Cátedra, 1998). 
6. Lector. Ante ideas como estas, Umberto Eco se ha dedicado en diversas ocasiones a precisar un modelo teórico de la interacción Lector-texto, expuesto la primera vez en 1962, en Opera aperta y posteriormente más formalizado en Lector in fabula, de 1979. Las ideas principales podrían puntualizarse así:

1. el texto puede considerarse "una inmensa máquina de producción de interpretaciones", pero al mismo tiempo también indica determinados recorridos de lectura ${ }^{6}$. De esta manera, existen interpretaciones buenas e interpretaciones malas o equivocadas, que hacen que no todas sean iguales;

2. ante una multitud de significados posibles no referenciales, un mensaje no puede decir cualquier cosa puesto que existen sentidos que no se pueden sugerir. En teoría, sobre un texto se pueden hacer infinitas conjeturas interpretativas. Pero al final, las conjeturas se probarán sobre la coherencia del texto y la coherencia textual no podrá más que desaprobar algunas conjeturas desatinadas. Esto sucede porque la participación del Lector no elimina el hecho de que el texto posee una estructura semántica y que esta conduce a varios tipos de isotopías textuales;

3. el lector participa enel proceso de interpretación textual, pero no 'por la libre', pues aún desde antes de su participación existen ciertas constricciones ${ }^{7}$, como por ejemplo, una bastante obvia pero que generalmente se olvida, que es la existencia de significados literales dentro de una lengua: esta restricción preliminar hace que "cualquier acto de libertad de parte del lector puede venir después y no antes de la aplicación de esta restricción"

4. si bien existen textos abiertos $y$, en general, los textos pueden suscitar lecturas infinitas, estas no pueden ser cualquier lectura:

6. Eco, 106.

7. Eco, 13.

8. Umberto Eco, I limiti dell 'interpretazione (Milán: Bompiani, 1990) 9. 
Es imposible decir cuál es la mejor interpretación de un texto pero es posible decir cuáles están equivocadas. En el proceso de semiosis ilimitada es posible ir de cualquier nudo a cualquier otro nudo pero los pasajes están controlados por reglas de conexión que nuestra historia cultural ha legitimado de alguna manera 9 .

Por su parte, en el libro de ensayos referido, Tzvetan Todorov considera que acusa al desconstruccionismo de escepticismo, fundamentalmente porque plantea que el conocimiento y el juicio de valor sobre la literatura son imposibles; también lo considera de posición dogmática porque "decide 'a priori"' que todo texto sólo dice nada ${ }^{10}$. Además, Todorov critica la idea del pragmatismo de que es cada lector quien otorga el sentido al texto. El teórico búlgaro considera que de esta manera se hace inútil la interpretación y el texto queda reducido a una especie de "test" de escogencia para los lectores ${ }^{11}$.

7. Teoría/interpretación. Mignolo. Se vuelve ineludible entonces tratar de distinguir el quehacer teórico del trabajo interpretativo. En el libro titulado Teoría del texto e interpretación de textos, Walter Mignolo propone que la interpretación es la "comprensión hermenéutica de la literatura" 12 , encargada de elaborar "inferencias sobre el sentido de una obra a partir de los principios generales de la literatura establecidos por la comunidad interpretativa"13. Estos principios generales son el conjunto de las definiciones esenciales de la literatura, las cuales se infieren de las normas de producción e interpretación del texto, es decir, los distintos conceptos de literatura que se suceden a lo largo de la historia de la literatura ${ }^{14}$, como literatura romántica, barroca, de vanguardia, etc.

9. Eco (1990), 107.

10. Tzvetan Todorov, El hombre desplazado (1996), edición en español (Madrid: Santillana, 1998) 229.

11. Todorov, 230.

12. Walter Mignolo, "Comprensión hermenéutica y comprensión teórica", Teoría del texto e interpretación de textos (México: Universidad Nacional Autónoma de México, 1986) 32.

13. Mignolo, 53.

14. Mignolo, 52. 
Para Mignolo, el trabajo interpretativo desborda las teorías debido en parte a que estas se dedican a estudiar aspectos parciales. Las teorías "no dan cuenta de un referente —el fenómeno literario, común a todas las teorías - sino de un objeto teórico" 15 , que es el objeto modelo, propio de cada teoría. Veamos dos casos, uno, el de Gérard Genette de Figuras III de 1972 y su revisión de 1983, cuya propuesta se ha utilizado muy a menudo como método para analizar la narrativa. El modelo de Genette concibe el texto narrativo en tres partes: la narración, el relato y la historia; en la edición crítica de 1983 aclaró la diferencia entre diégesis, entendido como 'universo', y la historia, como 'cadena de acontecimientos'. Ahora bien, Genette se dedica principalmente a estudiar la narración (o discurso) y el relato, y deja por completo abandonado el nivel diegético. Pero lo que ha sucedido en la práctica es que muy a menudo se toma este modelo como si fuera un método más de análisis, equivalente a cualquier otro, sin tomar en cuenta esta particularidad.

Algo similar ocurre con algunas teorías sociocríticas, que también se desentienden del estudio del plano de la historia - acontecimientos, personajes, espacio y tiempo-, e incluso de la figura del narrador, para concentrarse específicamente en el plano del discurso. Esto ocurre porque de acuerdo con algunas versiones del modelo sociocrítico el texto literario se entiende fundamentalmente como un objeto lingüístico, y por lo tanto, hay un interés particular en el estudio del discurso.

Si el interés es la interpretación de un cierto texto, no se puede recurrir a un modelo teórico como los anteriores, básicamente porque no abarcan la totalidad de la realidad textual. Si, por el contrario, el estudio se centra en aspectos de tipo teórico como la tipología del narrador, o bien, la relación entre texto literario y texto social, entonces es perfectamente legítimo apelar a marcos teóricos como los mencionados.

15. Mignolo, 42. 
Por ejemplo, si el interés de una investigación consiste en mostrar que no existe ningún rasgo que justifique una relación entre el sexo del escritor y el sujeto del enunciado, estamos en plena investigación teórica, dentro del campo de las teorías del sujeto de la enunciación. Aquí se pueden utilizar textos para comprobar la hipótesis, que es teórica, pero no para interpretar estos textos. Las conclusiones, entonces, se referirán a aquella relación, no a los posibles sentidos textuales.

Existen diversas teorías porque se estudian diferentes aspectos del fenómeno literario; por esta razón el objeto de estudio de cada teoría es diverso, ya que el objetivo de su trabajo es distinto. He aquí por qué existen diversos modelos teóricos: cada uno se propone estudiar un aspecto distinto del referente "literatura", y así construye un MODELO u objeto teórico diverso.

No se trata de no "utilizar" las teorías; se trata, primero, de no confundir la interpretación con la teorización. En segundo lugar, cuando se trabaja teóricamente, el objetivo no es "aplicar" las teorías ya construidas si no hacer teoría, teorizar. Las teorías no se construyen para ser aplicadas a los textos: estos, por el contrario, se utilizan para contrastar la validez de las hipótesis empíricas de los modelos teóricos.

En consecuencia, en términos estrictos no podría afirmarse que existe un objeto denominado 'literatura': desde la perspectiva teórica, existen diversos objetos teóricos en las distintas propuestas, uno cuando se trata de saber cómo se produce la significación, en la investigación teórica; y otro cuando se trata de saber cuál es la significación de un determinado texto en la interpretación.

Ecoinsiste en la distinción entre uso e interpretación de un texto, cuando explica que la función propia de un enunciado es significar; mencionar y referirse, la verdad y la falsedad son funciones del uso de un enunciado: "mencionar o referirse no es algo que hace una expresión, es algo que alguien puede hacer usando una expresión" (278). De esta manera, las teorías usan los textos, no los interpretan.

Para Todorov, la pregunta sobre la significación textual sigue siendo "buena pregunta": "y siempre hay que intentar responderla, sin 
volcarse en exclusiva sobre los contextos, históricos, estructurales u otros" (240). Por esto es que su reflexión sobre estas teorías conduce a una visión de carácter más ético e ideológico: después de analizar la posición de los marxistas norteamericanos y la posición de Paul de Man, critica la negativa de la interpretación, la falta de reconocimiento de diferencias entre hechos e interpretaciones, y la consideración de toda forma de conocimiento únicamente como discurso. Todo esto revela a Todorov no sólo un relativismo ante los valores sino una posición totalitaria.

8. Todavía queda otro aspecto de la interpretación que puede contribuir a complementar la distinción con la teoría; se trata del aspecto 'social' de la profesión. En Los límites de la interpretación dice Eco lo siguiente con respecto a la interpretación: "La actitud hacia los textos refleja una actitud correspondiente frente al mundo externo. Interpretar significa reaccionar al texto del mundo o al mundo de un texto produciendo otros textos" (325).

Esto resulta congruente con la crítica que hacen Greimas y Gadamer a la negativa de Derrida de la interpretación, que según ambos, proviene de una incapacidad - filosófica - de reconocer al Otro. Desde la perspectiva del semiótico francés, con el concepto de la "diferencia", Derrida permanece en el nivel de la percepción y el de la negación del sentido del mundo, pero no llega al momento de la aparición del juicio. Este es, precisamente, el gestoque funda, "la negación de lo negativo" y al mismo tiempo, lo que "hace aparecer la positividad".

Reconocido como el pensador que más ha contribuido al estudio contemporáneo del tema de la interpretación, Gadamer considera que tanto Derrida como Heidegger "se interpretan en realidad a sí mismos y no lo que respectivamente creen interpretar" (87). En relación con la actividad interpretativa explica además:

comprender equivale a comprender de otra manera. Lo que se retarda, lo que se desplaza cuando mi palabra alcanza a un 
oyente, y más cuando un texto alcanza a su lector, no se puede concretar jamás en una identidad fija. Allí donde se quiere que tenga lugar el comprender no puede haber nunca sólo identidad. Comprender se refiere más bien a que uno es capaz de poner en el lugar del otro y expresar lo que ha comprendido y qué es lo que tiene que decir sobre ello. Y esto significa precisamente no reproducir sin más sus palabras (77).

Gadamer hace una enriquecedora comparación de la actividad hermenéutica con la conversación. Analizando los conceptos claves de Derrida — la diferencia, la huella - explica que las preguntas y las respuestas propias del diálogo permiten que se manifieste "la alteridad de lo verdadero" (93). Agrega, además: "Pensar es diferenciar. Esto significa considerar lo uno y lo otro (...) la pregunta nos pone en la situación de tener que decidir entre posibilidades" e implica tomar una decisión: es más difícil hacer preguntas que dar respuestas (101-102). En el diálogo, añade Gadamer, ser interlocutor es una identidad que hay que ganarse durante una conversación, no es una identidad ganada de antemano: "la identidad va con la conversación (...) todo pensar es un diálogo consigo mismo y con el otro" (96).

9. Conclusión. La necesidad de la interpretación se funda, pues, también, en el inevitable diálogo con el otro, el texto, más allá del placer íntimo e individual del analista que logra descifrarlo. En el encuentro con lo diverso que le puede oponer la distancia histórica o la cercanía temporal, el texto se descubre en esta otra dimensión que no sólo puede lograr revelar a su Autor - su cultura, su época, sus ideas - sino también a su posible interlocutor. La interpretación es una actividad semiótica que se realiza entre seres humanos, es social. Interpretar un texto af ecta la relación entre la gente, y no interpretarlo también porque permite la inmovilidad, la ausencia de cambio. El antiintencionalismo radical significa un aislamiento, una marginalidad social, una falta de interacción con los demás. 
La interpretación no sólo es posible, es útil y necesaria. Hace falta conocer la estructura de los lípidos y la historia del cerdo, sí, pero también es preciso saber preparar los chicharrones para invitar a los amigos. 